\title{
Developing standards for institutional ethics committees: lessons from the Netherlands
}

$\mathrm{H} \mathrm{H}$ van der Kloot Meijburg and R H J ter Meulen Ethi-Call Consultancy for Institutional Ethics Committees, the Hague, and Institute for Bioethics Maastricht, the Netherlands, respectively

\begin{abstract}
This article presents standards for setting up and educating institutional ethics committees (IECs). These standards are based on experiences in the Netherlands, where IECs have been established in a large number of health care institutions. Though the IEC has become a generally accepted institution within Dutch health care, there are concerns over its effectiveness regarding the improving of the moral quality of clinical decision making. Health care practitioners and members of IECs too, experience a gap between the IEC and the reality of the clinical environment. At this moment, there is interest in developing programmes which educate practitioners in moral issues and how to deal with them, using the method of a structured debate on the ward. The IEC will not be made obsolete by this development, but can play a guiding role in the implementation of such programmes. Current concerns are the lack of patient representation in the Dutch IEC, and the loss of contact with the local community of health care practitioners because of the merger of hospitals into bodies similar to UK National Health Service (NHS) trusts.

(Fournal of Medical Ethics 2001;27 suppl I:i36-i40)
\end{abstract}

Keywords: Institutional ethics committee; ethics education; moral debate

\section{Authors' note}

In this article we will use the term "hospitals" to refer to the different type of health care institutions mentioned here.

\section{Introduction}

Over the past decade institutional ethics committees (IECs) have become generally accepted in the Netherlands. ${ }^{1}$ Since the eighties, an increasing number of IECs have been established by various health care institutions. These committees serve multiple purposes, whether they be active in general hospitals for acute care, nursing homes, institutions for the physically and mentally handicapped or psychiatric hospitals. Their assignment may be limited to one particular task or to a specific theme, for instance consulting with staff about complicated cases, or providing the hospital management with ethical advice on institutional policy such as palliative care. Alternatively, the ethics committee may have as its sole task the raising of moral awareness among employees in general, thereby enhancing the moral quality of the service. However, most IECs in the Netherlands have multiple responsibilities, combining a number of the tasks mentioned above.

As well as IECs, there are a considerable number of research ethics committees. It is a legal requirement that the ethical aspects of clinical trials are discussed and commented upon by institutional review committees. ${ }^{2}$ In order to be legally recognised, these committees must comply with strict legal standards regarding the disciplinary background of the members, the operating procedures and the number of protocols to be reviewed on an annual basis. At this moment, 78 review committees have been officially acknowledged by the central committee. ${ }^{3}$ Many of these committees combine the review of research protocols with the tasks of an IEC, as mentioned above. However, the review of protocols is usually very time-consuming so many of these so called "mixed" committees have little or no time to fulfil the tasks of an IEC. The guidelines laid down by the the recent law on medical research compelled many "mixed" committees, particularly those in smaller hospitals, to give up their task of a review committee for medical-scientific research protocols because they were no longer able to comply with the new requirements. For example, many of them were not able to appoint a methodologist, which is one of the requirements of the new law. Thus, currently, the large hospitals (including the academic hospitals) have a separate IEC as well as a research review committee, middle-sized hospitals have a "mixed" committee, combining research review and clinical ethics, and smaller hospitals (if they decide to have an ethics committee at all) tend to have only an IEC.

Except for the research review committees, there are no recent figures of the numbers of ethics committees. In 1992, in a survey of the federal health care organisations 234 hospitals were reported to have an IEC. ${ }^{2}$ This was $33.7 \%$ of all the health care institutions in the Netherlands $(n=434)$. There are no hard figures on the number of IECs at this moment. However, it is estimated there are a few hundred ethics committees, most of them being IECs. One reason for the rapid increase in the number of ethics committees might be the increasing influence of disciplines other than medicine in 
clinical decision making. The ethical quandaries, which in part could be attributed to conflicting disciplinary perspectives, needed a platform where they could be debated and resolved. As well as this internal process, there are external processes contributing to the rapid increase of IECs in the Netherlands. One important societal development is the increased number of government regulations, legal standards (for example regulations in regard to compulsory treatment), and professional guidelines. The ethical aspects of these measures needed a forum where they could be translated into guidelines for an institution, and where practitioners could ask for advice in individual cases related to these measures. Another external influence is the Dutch culture of consensus, also called the "polder model", implying that once confronted with a complicated issue an ethical committee may indeed be the appropriate framework in which to consider the dilemma at hand. Ethics committees provide society at large and the health care sector in particular, with a forum where professionals, in search of some form of consensus, are able to debate the ethical aspects of issues that arise in the arena of health care

In this contribution we will provide the reader with information concerning standards for education and the process of the setting up of an IEC. At the end we will draw some conclusions regarding the developments in the Netherlands.

\section{Setting up the IEC}

Here we will focus mainly on the preparatory phase of setting up an ethics committee. ${ }^{4}$.

Before one can make the decision to embark upon the establishment of such a facility a strategic plan is needed. The plan should contain a road map of how to proceed, and include matters such as an assessment of local circumstances and current conditions. Ethics committees should not come into being simply because someone, one day, decided that it was a "good thing" to have one, or because there happened to be some ethical emergency that needed acute attention. This approach is analogous to running before one has learned to walk. One can almost guarantee that this type of initiative will be short lived. Setting up an institutional ethics committee takes time and an amount of effort, as we will illustrate below.

One of the questions that requires an answer in the early stage is: "What kind of facility do people within our organisation envisage?" Roughly there are two models from which to choose.

The first model is a hierarchical model. One can opt for an ethics committee which functions as an ethical expertise centre. Complicated and difficult matters are turned over to the committee for advice. The committee is well equipped for the task, because all the available expertise is drawn into it. Under these circumstances the most likely consequence is that the committee will share its wisdom with the organisation. It will issue either its own, or on request, statements about particular situations that arise within the hospital community.
In this situation it is the norm for the committee to work "top down" and its activities are policy orientated. In general people will not be tempted to challenge the insights of this committee because of its expertise and professionalism. Given local circumstances this may be a realistic option.

The other option is to organise an ethics committee "bottom up". Committee members team up with employees and make themselves available wherever and whenever there is a need for ethical consultation. The focus is to help caregivers understand their own motives for the decisions they make. By discussing the issues, those involved add another dimension to the deliberations, the ethical dimension. During the process of discussing this ethical dimension, one can often be helped to understand either one's own, or others' reluctance to a presented solution. The outcome often provides the caregiver with alternatives not previously thought of. A side effect of this model is that caregivers often find that the heightening of their moral awareness becomes a valuable personal experience. In this scenario the endeavours of the committee members enhance ethical awareness throughout the organisation. One may not be able to pinpoint the spin-off, no documents or guidelines are produced, but it is all happening in the minds of the people who work in the institution. In contrast with the first option this choice can be very time-consuming, but it is well worth the effort.

The choice made determines the tasks and the composition of the ethics committee. The most common tasks are in the area of case consultation; raising the moral sensitivity of personnel (education); commenting on the ethical aspects of (existing) protocols and guidelines; advising the organisation on ethically sensitive policy issues, and organising the institutional debate on specific topics or themes (the "platform" function). Reading this list one is able to understand why some hospitals prefer a "top down" approach, while others think that a "bottom up" approach will suit their purposes best. The choice will also determine the committee membership. If the organisation thinks expertise is most needed the membership will differ from a situation where close contact and communication with the work-floor is prominent.

\section{Membership}

There are no legal or even general rules as to membership of the IECs. Generally membership consists of one or two physicians, a psychologist or psychiatrist, one or two nurses, a pastor, an ethicist, a lawyer and one or two paramedics. There is one person not mentioned here-the patient. Though the IEC is expected to protect the interests of the patient, IECs in the Netherlands never involve the patient or client directly. Statistics in the Netherlands are very consistent in showing that patient participation is practically non-existent in ethics committees. ${ }^{2}$ The reality is that committees serve the members of the hospital community first. However, one may argue that patients benefit indirectly, because the quality of the decision making process 
is enhanced by looking at the ethical implications of different treatment options.

General acceptance of such a facility by employees is essential for an ethics committee to survive. This raises the issue of professional responsibility versus the work of the IEC. Practitioners argue that professional standards require each individual health care provider to account for the ethical aspects of the decision he or she has to make and that these decisions could never be altered by additional insights or advice from ethics committees. This misrepresents the aim of ethics committees, which is not to replace the professional responsibilities of the individual caregiver but to enhance the quality of the decision making process, leaving the responsibility for the final decision to the professional.

More complicated, however, is the issue of conflicting interests among various groups of professionals, leading to resistance to a facility for ethical consultation. This is often related to traditions within the organisation and with the balance of power between different groups. Methods of communication are important: whether communication between different groups is strictly hierarchical and formal, or whether people are open to more casual forms of communication. Some may claim specific knowledge and impose their opinions on others on the basis of that knowledge, causing others eventually to lose heart. These circumstances may easily jeopardise the moral debate before it even has a chance to get under way. In this respect starting an ethics committee could constitute a significant challenge to the prevailing culture within the hospital environment. Starting such a committee needs a considerable amount of thought, effort and above all commitment.

\section{Management}

From the beginning those seeking to establish a committee should seek support for their initiative from the senior management of the organisation. The authoritative head (the president of the board or the chief executive officer) should be aware that the initiative to establish an ethics committee is his/ her responsibility. The management may commission a staff member to look into the matter more thoroughly. If the management fails to take this initiative then a member of staff could take the lead on behalf of the organisation, turning responsibility over to the management as soon as possible. In any case it is very important that the management endorses the need for ethical consultation and provides for it. An ethics committee should be part of the institution's policy regarding the way the organisation intends to cope with the ethical quandaries that may arise. Activities that issue from this policy should be made public and should also be an integral part of the annual report of the organisation.

The hospital administration is also responsible for creating the conditions under which the ethics committee can thrive. They do that by making the work of the committee a legitimate and integral part of the hospital services. In an organisation like a hospital acknowledgement of a committee's existence by the management is vital. This can be made explicit by facilitating the ethics committee activities, for example by providing the committee members with time to do their work. The management should also budget for a secretary to take notes, to convene the meetings and to provide clerical support in general. If necessary it should provide the committee with expertise in the field of health law and health care ethics, and with an adequate education and training programme. A hospital cannot maintain an ethics committee in its "spare" time. This kind of investment requires a real policy decision. The institution may have to prioritise ethical consultation over and above other items. Consequently, if an organisation decides to establish an ethics committee it must do so on a long term basis, otherwise it will be a waste of resources and a disappointment for all those who participate.

\section{Setting standards for educating the IEC}

Once started, the members of the IEC often realise they lack the necessary skills to analyse ethical issues and to set up a structured debate. Training courses have helped committee members to get a better grip on the issues at hand. The priority is to make employees aware that "doing ethics" is not something new. On the contrary, as individuals, we have already developed our own ways of dealing with ethical quandaries. Some practise ethics by applying professional standards, others do it on the basis of their experiences and their moral intuitions. Some do ethics on the basis of what their organisation has stood for over time, others will rely on the values and norms that have been handed down to them from when the organisation was still in its infancy. Consequently, one of the main aims of any educational programme is to explain to the committee members that practising ethics together is less difficult than is often thought. Secondly, during the process of moral deliberation, committee members benefit each other and the organisation by clarifying those (professional or personal) norms and values which lie at the heart of their moral positions.

However, to organise a structured ethical debate takes more than a clarification of the personal and professional values of committee members. What is required then, is a theoretical framework to guide the moral debate among the members, enabling them to weigh the different moral values at stake in a specific case or protocol. In the training courses organised by the Institute for Bioethics for instance, we begin with a presentation of some of the main theoretical concepts in health care ethics, illustrated by practical examples and cases. It is important that such a theoretical introduction does not go too far above the practical level of the clinic. On the whole health care practitioners are not interested in a thorough introduction to the philosophy of Immanuel Kant or John Stuart Mill. What they do need are practical tools for ethical analysis, for example, the distinction between "positive" and 
"negative" autonomy or criteria for incapacity. The "principles" approach, often despised for its abstract and rational nature, is often very helpful in structuring discussions on ethical dilemmas and in interpreting and weighing the values involved.

After this theoretical introduction, trainees are offered a step-by-step approach to analysing cases. This approach teaches them how to make the moral aspects of a case explicit (as distinct from communication problems or other non-moral problems), to analyse the main moral values that are involved, to weigh conflicting values in the case of a dilemma, and to formulate options for resolving the conflict. A similar analytical approach can be applied to drafting guidelines and protocols, such as "do-notresuscitate" protocols or protocols on organ donation. Committee members find such a step-by step approach very helpful in structuring their own individual thought processes. It also provides them with a mutually shared framework for debating various topics.

Another area of focus is teaching committee members about the advantages of a multiprofessional approach to ethical quandaries. Today IECs thrive because the whole approach to health care delivery has changed. The days have gone when the daily practice of health care meant one decided for all. Nowadays the team approach is paramount throughout the organisation. It has amounted to a profound change of culture. Teamwork implies the contribution of various disciplines important in preparing for, or evaluating, the course of action. A truly multiprofessional approach is based on professional respect for each individual contribution. There are cure-oriented solutions to a problem, but there may also be careoriented solutions to the same problem. To minimise the effects of pain one may advocate a step up in the administration of pain treatment, while others may suggest music therapy. Likewise, ethical insights and solutions offered may come from different professional perspectives.

Finally, committee members need to learn about the way modern organisations like a hospital operate. There seems to be a gap between the ethical way of thinking and speaking and that of the health care institutions at large. Attention has to be given to the kind of language and methodology organisations use when announcing their intentions and outlining their goals. Ethics committees are sometimes perceived by management to be occupying themselves merely "talking", implying that these committees are not adding much benefit to the hospital. An ethics committee which wishes to be effective will aim at conforming to the present institutional "way of life".

Looking back and concerns for the future

The IEC has become a generally accepted phenomenon in the Dutch health care setting. The majority of Dutch health care institutions have a committee for ethical advice on individual cases, for the development of policies and for educational activities on ethical issues. Now that the IEC has become a well established institution within the health care arena, it is time to look back and assess some of the outcomes for the future.

First of all the whole process has taken a considerable amount of time. The first ethics committee was established as far back as 1975! Apparently it is a facility that professionals have to get used to in order to incorporate it into their daily routine. Secondly, in the Netherlands we have had to learn not to underestimate the role of hospital management. Institutional ethics committees have no legal or federal footing, consequently, if management does not endorse the initiative it becomes extremely difficult for the committee to become firmly grounded within the organisation. The management helps to make ethical consultation within the hospital respectable by legitimising it, by providing for it and integrating it with hospital policy and services. This is a mutual responsibility, because in turn committee members should make the management aware of why the committee needs their support. Thirdly, an IEC can only thrive if it takes care of itself. Support from the management should be made visible in its providing the committee with training programmes and promoting the continual education of its members.

\section{Patient representation}

With regard to the future there are some concerns. Some have voiced their concern over the effectiveness of the IEC. $^{6}$ Does the committee really improve the moral quality of clinical decision making? Do doctors, nurses and other practitioners profit from the activities of the IEC in dealing with moral issues in their work? Practitioners, managers and even members of the IEC themselves, often experience a gap between the IEC and the reality of the clinical environment, where people are continuously exposed to moral dilemmas and often do not know how to cope. For this reason, there is an increasing interest in other methods and instruments to achieve the goals of the IEC. Moral awareness is needed on the wards, thus the ethical debate should take place among the practitioners themselves. Some managers prefer the methods associated with quality assurance programmes and doubt the necessity of a separate facility for ethical issues: they believe that such an integrated instrument is of more help to them when trying to analyse a case than advice from a "distant" committee. However, this development will not render the IEC superfluous. The committee itself can become instrumental in initiating and guiding the implementation of such instruments and programmes. Another concern is the issue of patient representation. Enhancing the quality of care by looking at ethical aspects is in the direct interest of the patient, so why is the patient not represented on the committee so that the patient can express an opinion? Is it acceptable that practitioners speak on behalf of their patients? Do caregivers always have the best interests of the patient in mind? One has to acknowledge that there is an imbalance and this may be a difficult problem to 
solve. We believe that both concerns need further exploration.

We have recently observed many health care organisations merging into big conglomerates, a development akin to the setting up of health care trusts in the United Kingdom. In the wake of these mergers local IECs have also merged, thus becoming estranged from their local surroundings. It has turned them into truly "distant" entities for ethical consultation. As communication lines grow longer IECs are faced with the problem of how to maintain contact with the practice of daily care. Some committees have developed sites on their organisation's web pages and can be consulted directly via the internet.

Change is an ongoing process. Institutional ethics committees came into existence because employees developed a multiprofessional approach to ethical quandaries in health care. Perhaps IECs will become superfluous and disappear as professionals discover new forms of communication regarding the moral dilemmas they encounter. In retrospect the acknowledgement that these dilemmas exist and that something can be done about them, would imply that, over time, IECs have made a significant contribution to the raising of moral awareness within the health care profession.

$H$ H van der Kloot Meijburg is Director of Ethi-Call Consultancy for Institutional Ethics Committees, the Hague, The Netherlands. $R H \mathcal{F}$ ter Meulen is Director of the Institute for Bioethics and Professor of Philosophy, Department of Caring Sciences, University of Maastricht, the Netherlands.

\section{References}

1 Kloot Meijburg HH van der. Different profiles for institutional ethics committees in the Netherlands. HEC Forum 1994:3:13956.

2 Wet medisch-wetenschappelijk onderzoek met mensen. Staatsblad. 's-Gravenhage: Sdu, 1998: 161.

3 Centrale Commissie Medisch-wetenschappeliijk Onderzoek (CCMO), www.ccmo.nl, 22-8-2000.

4 Kloot Meijburg HH van der. De ethische commissie: het voortraject. Tijdschrift voor Geneeskunde en Ethiek1996;6:15-22. 5 Willigenburg T van, Beld A van den, Heeger FR, Verweij MF. Ethiek in praktijk. Assen: Van Gorcum, 1993.

6 Verweij MF, Brom FWA, Huijbers AK. Ethiek in commissie. Utrecht: Nederlandse Vereniging voor Bio-ethiek, 1999. 